\title{
Immersive Radiation Experience for Interventional Radiology with Virtual Reality Radiation Dose Visualization Using Fast Monte Carlo Dose Estimation
}

\author{
1) Graduate School of Medical Care and Technology, Teikyo University, Japan \\ 2) Department of Radiology, Teikyo University School of Medicine, Japan \\ 3) Central Radiology Division, Teikyo University Hospital, Japan
}

Takeshi Takata ${ }^{1)}$, Hiroshi Kondo ${ }^{2)}$, Masayoshi Yamamoto ${ }^{2)}$, Kenshiro Shiraishi' ${ }^{2)}$, Takenori Kobayashi ${ }^{1)}$, Shigeru Furui ${ }^{122}$, Takahide Okamoto ${ }^{133)}$, Hiroshi $\mathrm{Oba}^{22}$, Jun'ichi Kotoku ${ }^{133}$

\begin{abstract}
For interventional radiology (IR), understanding the precise dose distribution is crucial to reduce the risks of radiation dermatitis to patients and staff. Visualization of dose distribution is expected to support radiation safety efforts immensely. This report presents techniques for perceiving the dose distribution using virtual reality (VR) technology and for estimating the air dose distribution accurately using Monte Carlo simulation for VR dose visualization. We adopted an earlier reported Monte-Carlo-based estimation system for IR and simulated the dose in a geometrical area resembling an IR room with fluoroscopic conditions. Users of our VR system experienced a simulated air dose distribution in the IR room while the irradiation angle, irradiation timing, and lead shielding were controlled. The estimated air dose was evaluated through comparison with measurements taken using a radiophotoluminescence glass dosimeter. Our dose estimation results were consistent with dosimeter readings, showing a $13.5 \%$ average mutual difference. The estimated air dose was visualized in VR: users could view a virtual IR room and walk around in it. Using our VR system, users experienced dose distribution changes dynamically with $\mathrm{C}$-arm rotation. Qualitative tests were conducted to evaluate the workload and usability of our VR system. The perceived overall workload score (18.00) was lower than the scores reported in the literature for medical tasks (50.60) and computer activities (54.00). This VR visualization is expected to open new horizons for understanding dose distributions intuitively, thereby aiding the avoidance of radiation injury.
\end{abstract}

Key words: radiation dose, Monte Carlo, virtual reality

(Interventional Radiology 2020; 5: 58-66)

\section{INTRODUCTION}

Risks of severe radiation injury to patients and staff members have recently increased concomitantly with the number of interventional radiology (IR) operations [1, 2]. The irradiation direction and dose differ considerably according to the IR procedure; therefore, interventional radiologists and other staff members can be exposed to harmful doses of ra- diation. The air dose distributions, which cannot be seen with the naked eye, also vary considerably in such cases. For these reasons, precise knowledge of the air dose distribution for each operation is expected to be helpful in protection against radiation.

Education and training related to radiation risk control for interventional radiologists and other medical staff are recommended in International Commission on Radiological Protection (ICRP) Publication 113 [3]. The lack of adequate ra- 
diation safety education has been reported in several earlier studies [4-6]. Conventional illustrations of dose distribution are difficult to understand intuitively because they are presented in 2D, even for data obtained initially in 3D.

Previous studies have examined the visualization of radiation dose distributions in an IR room. Bott et al. developed a computer-based training and simulation system [7]. Although this system includes a function that visualizes the scattered dose, the system visualizes it at the minimal range around a fluoroscopic target. This system does not incorporate necessary parameters of scattered radiation production (e.g., X-ray beam spectrum, field-of-view, C-arm angle, and patient body). Therefore, a precise dose visualization system is necessary to estimate the plausible scattered dose distribution based on the physical process.

Previous studies [8-11] have proposed dose visualization systems combining Monte Carlo dose calculation with augmented reality (AR) dose visualization. Monte Carlo methods are mainly used as dose calculation algorithms. Nevertheless, such methods require long durations to produce highly precise estimates. Long periods must be allowed for the simulation of all X-ray beams irradiated from various directions in IR. Dose calculation and visualization using lead shielding, which is widely used in clinical cases, were not proposed in earlier studies. Although AR can impart virtual information to the real 3D environment, users can only perceive information through the small $2 \mathrm{D}$ screen of a tablet device and monitor. As with traditional computer-based systems on a desktop computer, AR insufficiently encourages users to understand it intuitively. Loy Rodas et al. presented the possibility of its application to an AR system using a head-mounted display, but no specific method was described [9].

Virtual reality (VR) technology enables users to immerse themselves in a virtual world and to move freely around a real-scale 3D space. Immersive VR provides a physical geometric arrangement that lets a user grasp 3D structures. Some earlier reports have described use cases and benefits of VR training [12, 13]. For this study, we adopted VR technology to visualize the dose distribution dynamically in IR. Its use was intended to raise staff members', especially nonexpert staff members', understanding of the radiation dose related to IR. This report is the first in the relevant literature to propose an immersive VR radiation visualization system for IR- The Virtual Reality Dose Visualization System (VR-DVS). It is based on Monte Carlo dose simulation running on a graphical processing unit (GPU) [14, 15]. Using GPU calculation reduces calculation costs drastically, even for simulations that incorporate calculations for lead shielding.

This immersive system visualizes the patient skin and air dose distributions with an arbitrary irradiation angle. A user can observe changes in the dose distribution in a virtual IR room. As reported earlier in the literature $[12,13]$, VR-DVS can be expected to engender a marked improvement in education about radiation protection.

\section{MATERIALS AND METHODS}

Before VR visualization, air and skin dose distributions in IR were estimated using the fast dose estimation system for IR (FDEIR), which uses a GPU to estimate the dose distribution through Monte Carlo simulation. It suppresses electron transport to accelerate calculations [14]. Then, VR-DVS loads and visualizes these dose distributions in a virtual IR room. FDEIR executes the Monte Carlo simulation on a voxelized geometry constructed from an individual patient's computed tomography (CT) dataset. For this study, we replaced the patient CT geometry with the IR room geometry and estimated the air dose distribution.

\section{Air Dose Estimation}

Calculating the air dose distributions that occur under various fluoroscopic conditions demands tremendous computational resources. Therefore, a dose for VR-DVS must be computed before real-time visualization. Unfortunately, standard Monte Carlo packages are too slow for calculations that support ready visualization. In this study, we calculated the air dose using FDEIR [14], which uses GPU computing to simulate the absorbed dose very quickly.

\section{Framework of the FDEIR dose estimation system}

As described in an earlier report [14], FDEIR calculates the absorbed dose to the skin using Monte Carlo simulation. This system conducts a simulation using a patient's CT dataset with fluoroscopic conditions that include the tube voltage, energy spectrum, milliampere value, source-to-imagereceptor distance, field-of-view size, source position, and the imaging table position. In this study, the simulation works with the IR room geometry, and air dose distributions in the IR room are estimated. The simulated relative dose must be calibrated to estimate the absorbed dose. For this purpose, we applied a scaling factor for conversion of the relative dose in FDEIR to the absolute dose from an earlier study [14]. The scaling factor was acquired using the same fluoroscopic unit (Innova 4100-IQ; GE Healthcare Inc.) and under similar fluoroscopic conditions as that study [14]. The scaling relation was a linear regression equation of $y=0.203 x$ $\times 10^{11}$, where $y$ and $x$ represent the absorbed dose (Gy) and the simulated relative dose per photon (Gy), respectively. These computed air and skin dose distributions were then incorporated into VR-DVS.

\section{Verification of FDEIR air dose estimation}

The calculation precision of FDEIR under the skin dose estimation on the patient's CT dataset was verified as explained in an earlier report [14]. However, the air dose calculation precision was not verified. Therefore, we validated the precision in this study. We chose PHITS version 2.93 [16] as a reference to verify the FDEIR simulation. As a typical Monte Carlo dose simulation code, PHITS incorporates Electron Gamma Shower version 5 (EGS5) [17], for 


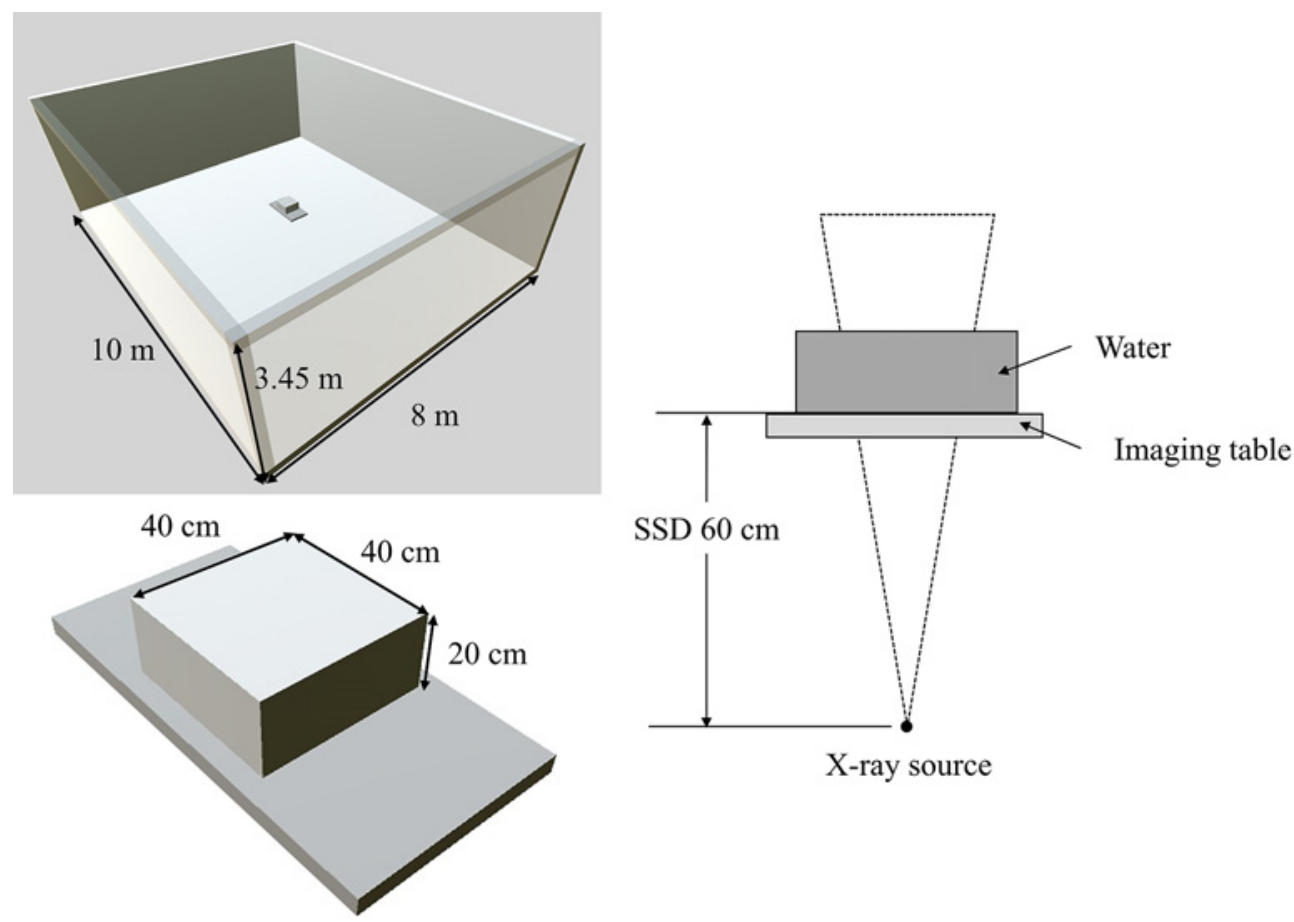

Figure 1. Simple room geometry for validation between FDEIR and PHITS.

which photon and electron transport have been well established $[18,19]$.

The FDEIR and PHITS simulations were conducted on a single Tesla P100 GPU (NVIDIA Corp.) and two central processing units (CPUs; E5-2695v4 Xeon; Intel Corp.) with 36 cores and 72 threads on a supercomputing system (Reedbush-H; Silicon Graphics International Corp.), respectively, at the Information Technology Center of The University of Tokyo, with simulation of 10 billion incident photons. The photon cut-off energy was $5 \mathrm{keV}$ in each case. The electron cut-off energy was $5 \mathrm{keV}$ in the PHITS simulation. These simulations were conducted with a simple geometry that included a water-equivalent phantom, an imaging table, air, and ordinary concrete walls of $20 \mathrm{~cm}$ thickness $(10 \mathrm{~m} \times 8 \mathrm{~m} \times 3.45 \mathrm{~m}$; Figure 1). The space was divided into mesh voxels $(5 \mathrm{~cm} \times 5 \mathrm{~cm} \times 5 \mathrm{~cm})$ because the electron range at the scatter photon energy range in air is $<5$ $\mathrm{cm}[20,21]$. Calculation times were recorded to elucidate the computational resources.

We also measured the air dose using a radiophotoluminescence (RPL) glass dosimeter (GD-352M; Chiyoda Technol Corp.) equipped with an energy compensation filter for lowenergy photons with a readout system (FGD-1000; Chiyoda Technol Corp.). The readout system is equipped with an internal calibration RPL glass dosimeter and a reference RPL glass dosimeter. A $1 \mathrm{~h}$ annealing process at $400^{\circ} \mathrm{C}$ was applied before exposing the RPL glass dosimeter to X-rays. Measurements were taken at horizontal distances of 50, 60, 70,80 , and $90 \mathrm{~cm}$ from the X-ray source at a height of 80 $\mathrm{cm}$ above the floor during $10 \mathrm{~min}$ fluoroscopy with a waterequivalent phantom (Figure 2) using Innova 4100-IQ fluoroscopic unit. Two RPL glass dosimeters were placed at

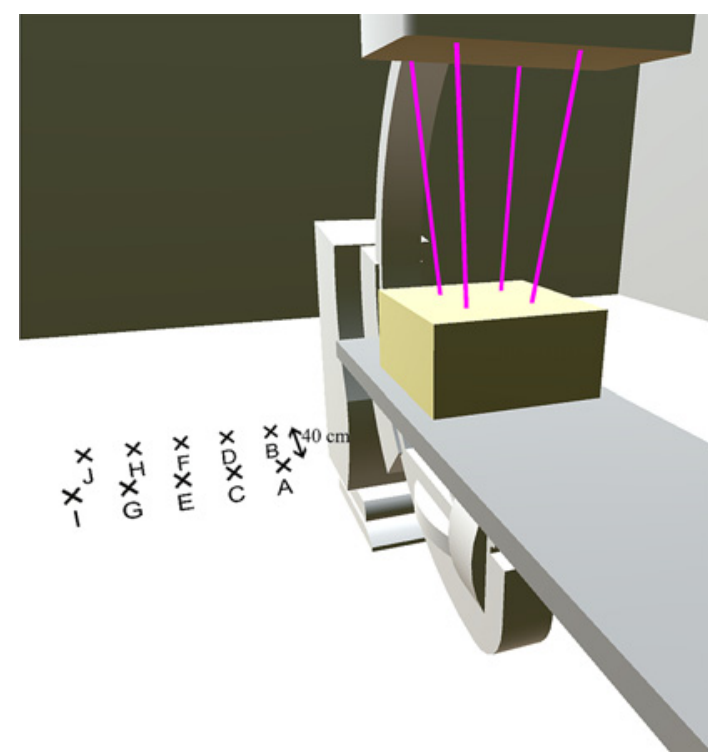

Figure 2. Simulated and measured points A-I. A, C, E, G, I and $\mathrm{B}, \mathrm{D}, \mathrm{F}, \mathrm{H}, \mathrm{J}$ were placed respectively at distances of $20 \mathrm{~cm}$ horizontally.

the same point. A 30 min preheating process at $70^{\circ} \mathrm{C}$ was applied before reading out the dosimeters. The readout system uses ultraviolet laser excitation to read the RPL signal. The average of two measured values was used for comparison. Those measured points corresponded to one standing position of the interventional radiologists and one place where scattered radiation can be measured efficiently. Other fluoroscopic conditions used for this study are presented in Table 1. These simulations were also conducted using FDEIR on a Tesla P100 GPU in the comparison geometry 
Table 1. Fluoroscopic conditions of the X-ray beam used for this study (Innova 4100-IQ).

\begin{tabular}{ll}
\hline Parameter & \\
\hline $\mathrm{kV}$ & $76 \mathrm{kV}$ \\
$\mathrm{mA}$ & $2.5 \mathrm{~mA}$ \\
Field of view & $20 \times 20 \mathrm{~cm}^{2}$ \\
Frames per second & 15 \\
Source to surface distance & $60 \mathrm{~cm}($ at PA) \\
Source to image-receptor distance & $100 \mathrm{~cm}$ \\
Filter & $0.3 \mathrm{mmCu}$ \\
\hline
\end{tabular}

\section{(Figure 2).}

\section{Simulation of IR room dose distributions}

To estimate the air dose, we constructed a voxelized geometry resembling an IR room $(10 \mathrm{~m} \times 8 \mathrm{~m} \times 3.45 \mathrm{~m})$ in an IR room at our institution. The geometry was sufficient to include a simple model of a fluoroscopic unit, a patient phantom [22], a suspended lead screen, a lead table shield, air, and ordinary concrete walls of $20 \mathrm{~cm}$ thickness. This unit model was based on public structure data (3D Warehouse; Google Inc.) for a carbon imaging table and an iron fluoroscopic unit. The suspended lead screen and the lead table shield had $2 \mathrm{~mm}$ and $0.5 \mathrm{~mm}$ lead-equivalent thickness, respectively, which we referred from the lead shields used at our institution. We treated the patient phantom as water for simplicity. The geometry was divided into mesh voxels $(5 \mathrm{~cm} \times 5 \mathrm{~cm} \times 5 \mathrm{~cm})$. The room geometry was also incorporated in VR-DVS.

We specifically examined a situation related directly to the risk of radiation dermatitis in the Cardiac IR where the $\mathrm{X}$-ray beam used for cardiac fluoroscopy was aimed at the chest of the patient phantom [22]. The air dose distributions were simulated at each irradiation angle. The $\mathrm{C}$-arm rotated $0^{\circ}$ to $360^{\circ}$ in lateral angle for virtual representation of an over-table tube, not only an under-table tube, and $-30^{\circ}$ to $30^{\circ}$ between the cranial and caudal angles, which are generic alternatives in cardiac IR. Additionally, a suspended lead screen and a lead table shield were placed to represent typical radiation shields. The fluoroscopic conditions are presented in Table 1, which is obtained from Innova 4100IQ with postero-anterior exposure. Dose simulations were performed assuming that the $\mathrm{C}$-arm was rotated under this condition.

A Monte Carlo simulation of 10 billion incident photons was used to estimate the air dose distribution voxels in the voxelized IR room using FDEIR. Skin dose distributions of the patient phantom were simulated at each irradiation angle on a geometry constructed from a CT dataset $(5.37 \mathrm{~mm} \times$ $1.074 \mathrm{~mm} \times 5 \mathrm{~mm})$.

\section{Building a virtual reality environment}

VR-DVS was originally designed for an immersive expe-

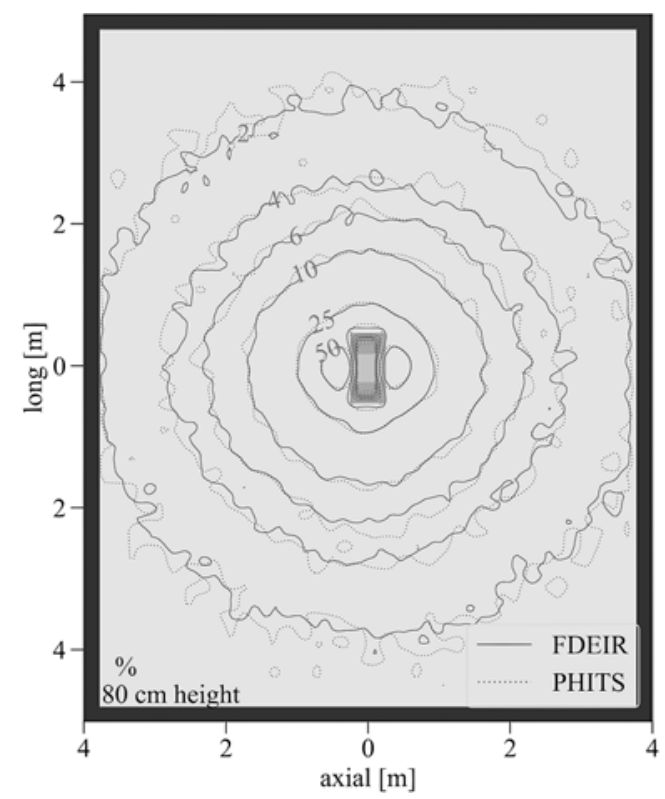

Figure 3. Relative dose distributions at $80 \mathrm{~cm}$ from the floor evaluated using FDEIR and PHITS. The distributions were normalized to the respective maximum doses.

rience of estimating air and skin dose distributions in IR operation using a real-time 3D graphics platform (Unity; Unity Technologies). We used a VR headset (HTC Vive; HTC and Valve Corp.) for the VR experience. Users of this VR headset have the sensation of walking around in a VR space. They can control the irradiation angle, irradiation timing, and the suspended lead screen and lead table shield via a wireless controller. In real time, VR-DVS loads and displays air and skin dose distributions from a stored dose table precomputed by FDEIR for visualization of the dose corresponding to fluoroscopic conditions.

In VR-DVS, users can also experience an eye lens dose, which presents an important difficulty for medical staff members. The energy spectra of scattering photons are completely different depending on the location in the IR room (Supplementary Figure 1). To prevent underestimation, the absorbed dose of the eye lens was converted from the air dose using the reported conversion factors $1.550 \mathrm{~Gy} / \mathrm{Gy}$ in ICRP Publication 74 [23], which is the highest value.

\section{Qualitative assessment}

This study examined 13 participants (2 radiologists and 11 radiological technologists) to assess VR-DVS. The NASA Task Load Index (NASA-TLX) questionnaire was used to evaluate the VR experience [24]. As a subjective workload assessment tool, NASA-TLX allows users to conduct subjective workload assessments of users. The NASATLX produces an overall workload score of 0-100 based on a weighted average of ratings on six subscales [24].

1) Mental demand: How much mental and perceptual activity was required?

2) Physical demand: How much physical activity was required? 


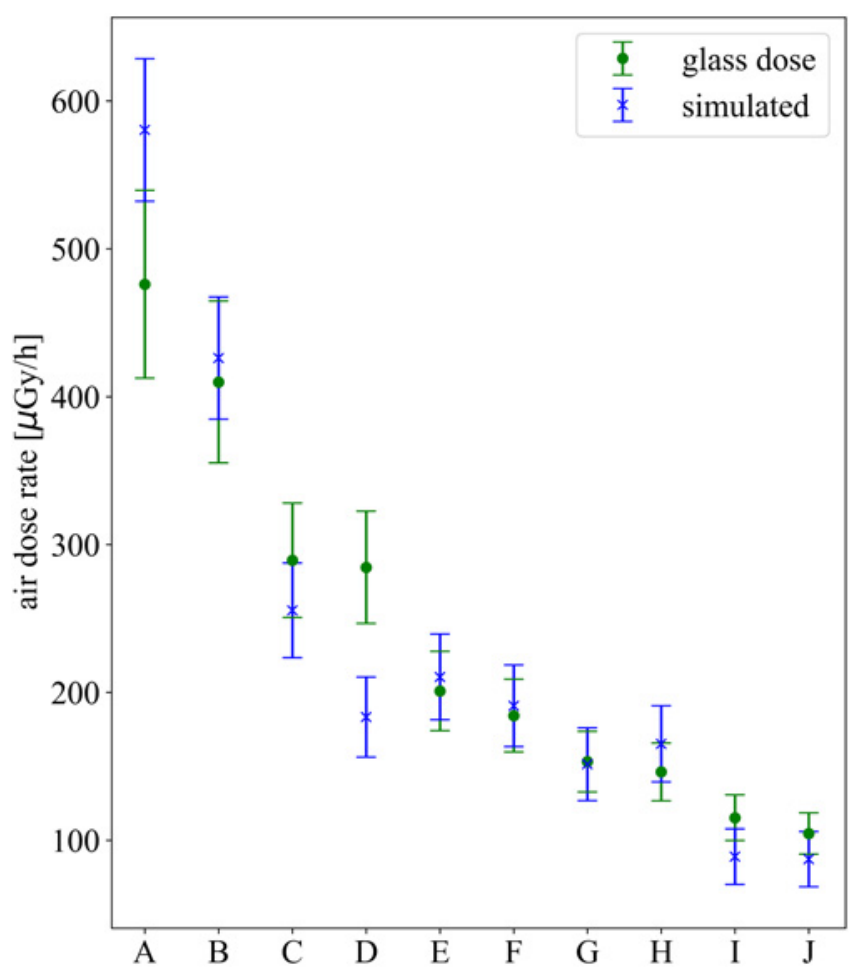

Figure 4. Absorbed doses for the simulated absorbed dose by FDEIR and measurements taken using RPL glass dosimeters. Error bars show standard deviations for FDEIR simulation $(2 \sigma)$, the propagation errors of variations in reading reproducibility (5\%), and the angular dependence $(12.4 \%)$ of the RPL glass dosimeter.

3) Temporal demand: How much time pressure did you feel because of the rate or pace at which the tasks or task elements occurred?

4) Performance: How successful do you think you were in accomplishing the goals of the task set by the experimenter?

5) Effort: How hard did you have to work to accomplish your level of performance?

6) Frustration: How insecure, discouraged, irritated, stressed, and annoyed versus secure, gratified, content, relaxed and complacent did you feel during the task?

The NASA-TLX Questionnaire was administered to identify the primary score of the workload during the execution of VR-DVS.

\section{RESULTS}

\section{Verification of the FDEIR-estimated air dose}

We compared the results obtained using a rapid and accurate dose estimation method explained in an earlier report, FDEIR, with those obtained using PHITS to verify the estimated air dose distribution. Figure 3 presents the air dose distributions calculated using those methods. The difference of the air dose in the room between them, [(FDEIR dose PHITS dose) / PHITS dose], was $15.2 \%$ on average at a height of $80 \mathrm{~cm}$. These results were mutually consistent, even if the electron transport was suppressed in FDEIR, implying that FDEIR estimates the air dose distribution reasonably well.

A simulation with 10 billion incident photons was completed within $3.6 \mathrm{~h}$ on FDEIR and within $9.5 \mathrm{~h}$ on PHITS under fluoroscopic conditions (Table 1). With a single Tesla P100 GPU, FDEIR achieved rapid air dose simulation in the IR room. The simulation results were consistent with data measured using RPL glass dosimeters by a $13.5 \%$ difference on average and a maximum of $104.2 \mu \mathrm{Gy} / \mathrm{h}$ (Figure 4). They were also consistent with the air dose measured by Lee under similar conditions [25].

\section{Dose distributions in the IR room}

We have presented examples of the estimated eye lens dose distributions incorporated in VR-DVS with and without the suspended lead screen and lead table shield (Figure 5). Comparison of the over-table tube and the under-table tube along a dotted line presented in Figure $\mathbf{5}$ without lead protection (Figure 6) reveals that the dose in the over-table tube was up to 24 times higher than the dose in the undertable tube. Similarly, comparing them with lead protection, the dose in the over-table tube was up to 8 times higher than the dose in the under-table tube.

\section{Virtual reality dose visualization}

A VR-DVS overview is presented in Figure 7 and Supplementary Video 1 and Video 2. Real-time skin and air dose distributions corresponding to the irradiation angle are depicted, respectively, as colored cubes at the skin position and colored spheres placed at intervals of $35 \mathrm{~cm}$ in the VR space. The sphere size and color represent the absorbed dose level. The absorbed dose increases proportionally with the exposure time. The eye lens dose of the user was also estimated using VR-DVS, which detects the head-mounted display position as the eye lens position. VR-DVS has the following five functions:

(1) Control of virtual X-ray irradiation, C-arm rotation, and placement of lead protection with a handy controller

(2) Visualization of the accumulation of air and the patient's skin dose distribution corresponding to the user operation

(3) Visualization of the air and the patient's skin dose rate distribution corresponding to the $\mathrm{C}$-arm rotation

(4) An indication of the user's eye lens dose at the detected head-mounted display position

(5) Visualization of scattered photon tracks corresponding to the $\mathrm{C}$-arm rotation

Users can walk around the virtual IR room and can learn the air and patient skin dose distribution corresponding to the $\mathrm{C}$-arm rotation at any place in the room. Function (1) enables intuitive operation for users in the virtual IR room without external operation. Function (2) visualizes accumulation of the dose distribution when X-ray beams are irradiated from various directions. Function (3) encourages intui- 
(a)

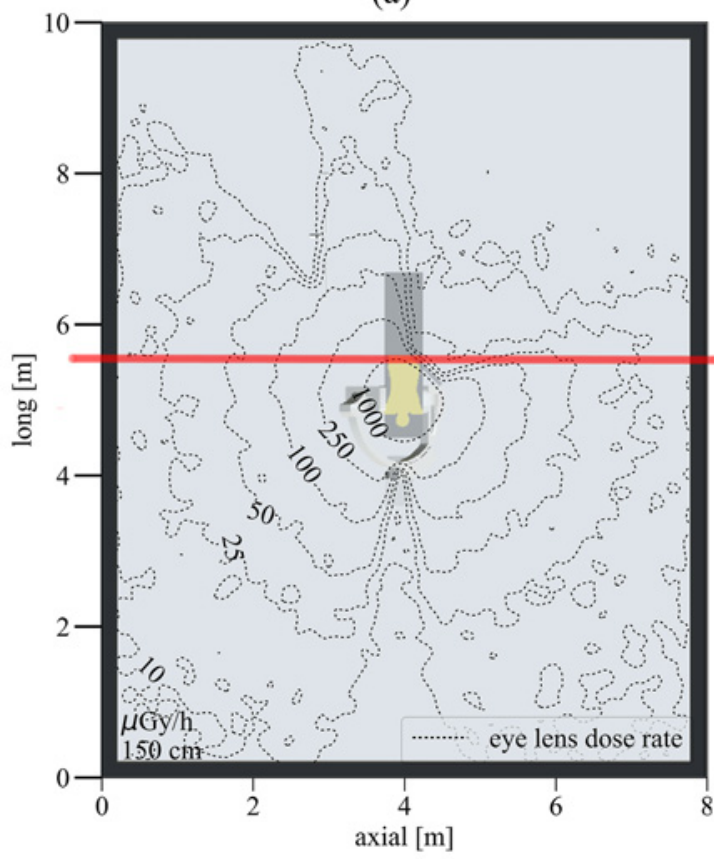

(c)

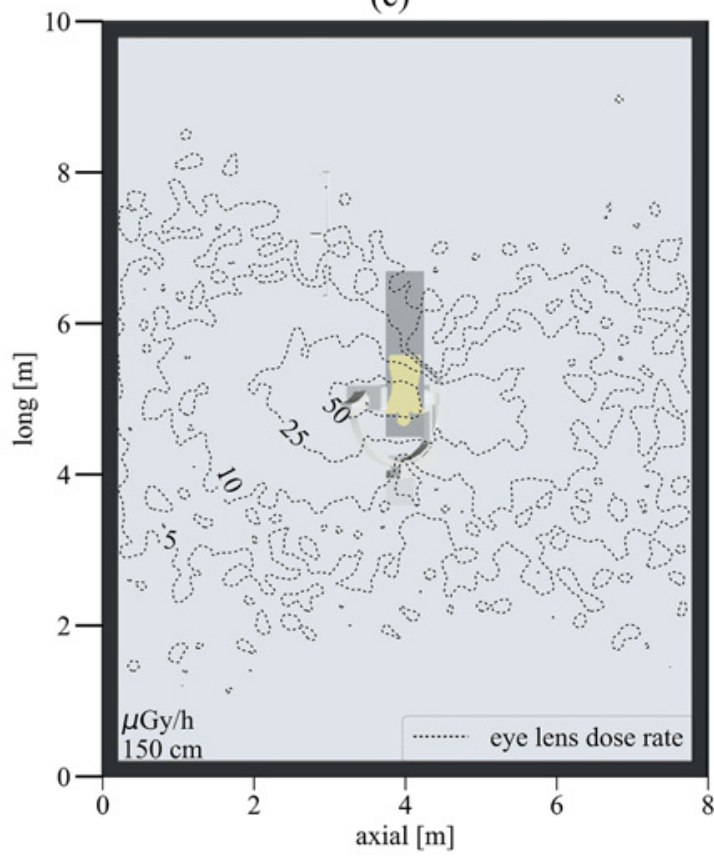

(b)

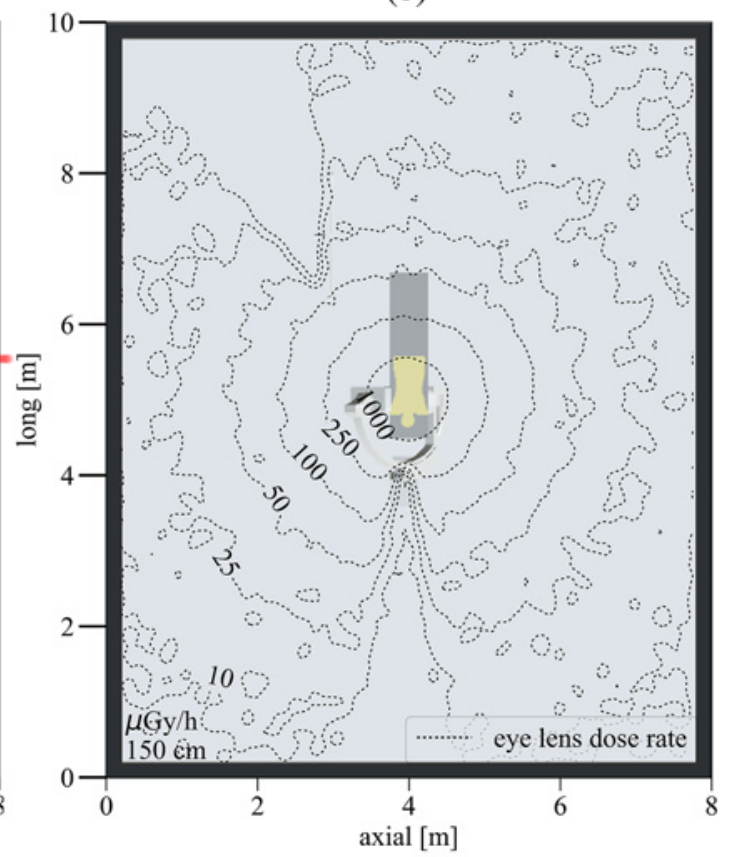

(d)

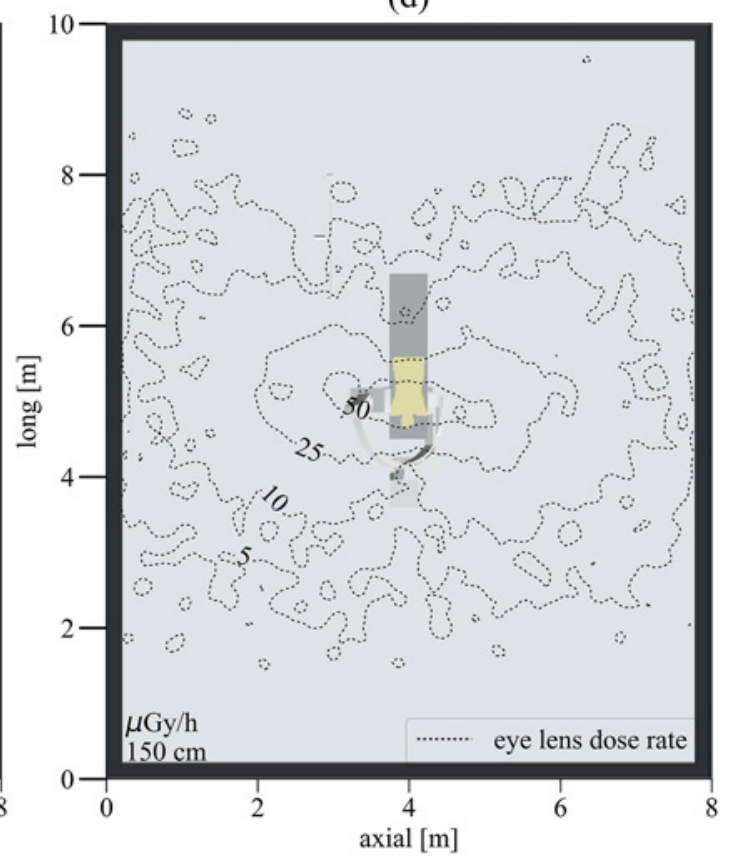

Figure 5. Eye lens dose distributions at $150 \mathrm{~cm}$ from the floor, estimated using FDEIR under fluoroscopic conditions (Table 1): (a) X-rays were irradiated in the over-table tube with lead protection, (b) in the over-table tube without lead protection, (c) in the under-table tube with lead protection, and (d) in the under-table tube without lead protection. Red lines represent suspended lead screens. Along with the dotted line on (a), dose distributions are presented for comparison in Figure 6. Lowdose areas at the 6 o'clock and 11 o'clock positions result from absorption at the fluoroscopic unit ( $\mathrm{C}$-arm and the suspended monitor). Statistical error resulted in the isodose curve shape.

tive understanding of a low dose rate area corresponding to the $\mathrm{C}$-arm rotation in the virtual IR room. Function (4) shows the pinpoint eye lens dose in addition to colored spheres filling the room. Function (5) shows scattered photon tracks to show the direction for radioprotection. Visualization of the radiation raises our understanding of the dose distributions.

\section{Qualitative evaluation}

Figure 8 presents results of the subjective workload scores from the NASA-TLX questionnaire. The median of the overall workload score (18.00) was lower than the scores for medical tasks (50.60) and computer activities (54.00) reported in the literature [26]. These results suggest that VR- 


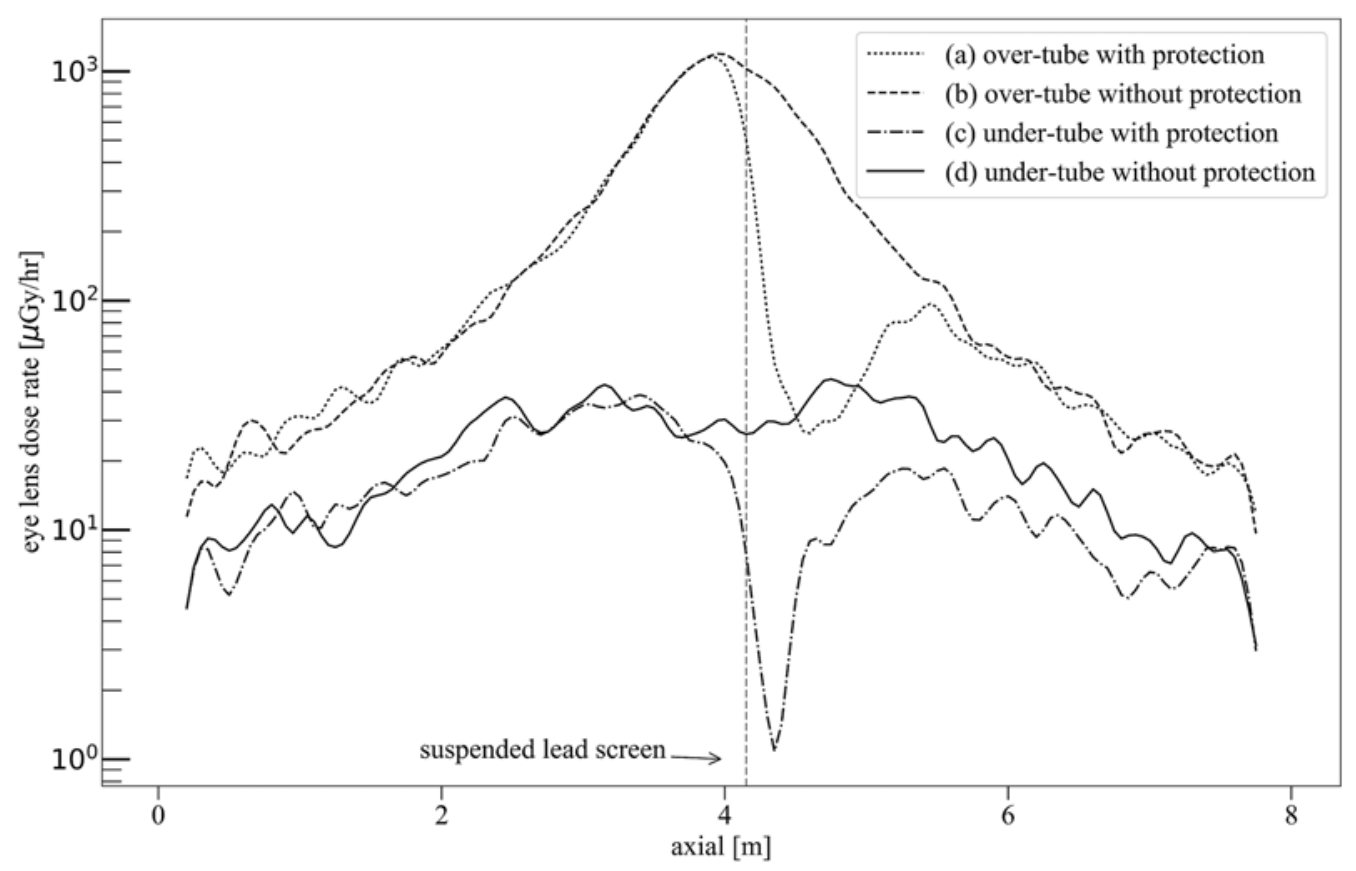

Figure 6. Eye lens dose distributions along a line presented in Figure 5.

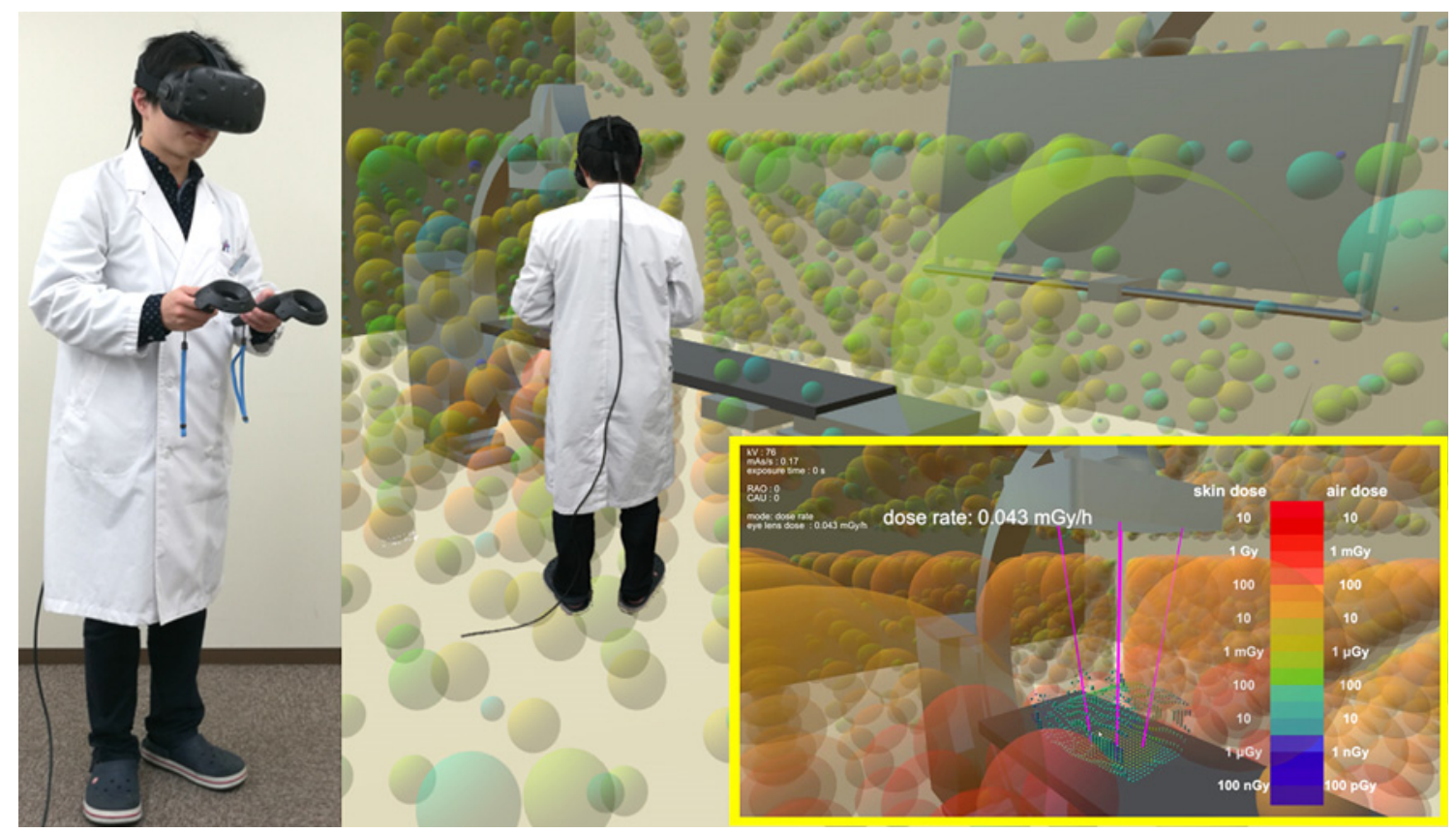

Figure 7. VR-DVS overview. The C-arm angle, X-ray exposure, and existence of the suspended lead screen and the lead table shield were adjusted using controllers. "Dose rate" in the inset shows the user's eye lens dose at the current position.

DVS can present dose information intuitively, thereby reducing the workloads of medical staff members.

\section{DISCUSSION}

We demonstrated the world's first immersive dose visualization system using VR technology, which provided a physical geometric arrangement that let the user view a 3D representation of the air and skin dose distributions. Furthermore, we proposed air dose estimation for IR using FDEIR while achieving rapid calculation and high precision for VR. This VR method is useful as an instructional tool when staff members must be trained about the radiation dose in the IR. Additionally, by identifying the standing positions of a radiologist during operations, detailed radiation exposure to the radiologist can be estimated rapidly.

The GD-352M RPL dosimeter has angular dependence for the radial direction and the long axis direction, respectively, of within $3 \%$ and $12 \%$ [27]. Only when the dosimeter is rotated 270 degrees in the long axis direction does the 


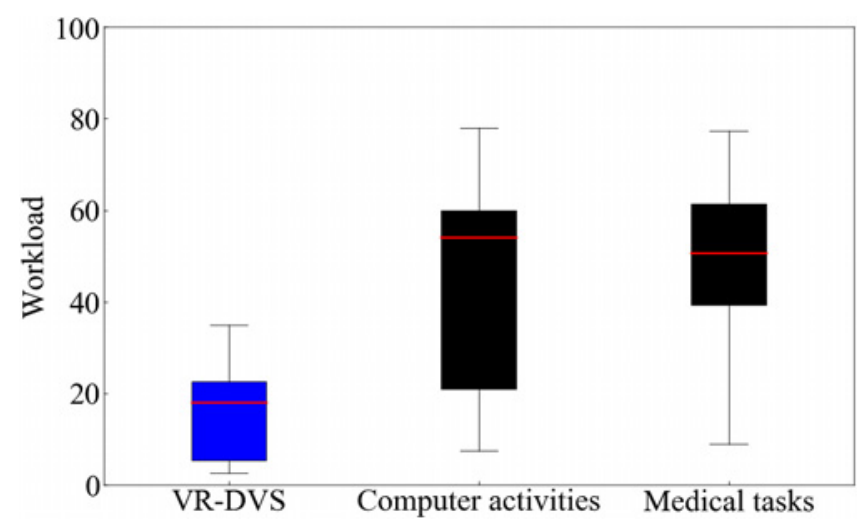

Figure 8. NASA-TLX workload score for VR-DVS (blue box) and scores for medical tasks and computer activities reported in the literature (black boxes) [26].

angular dependence become approximately 10\% [27]. However, all photon tracks reaching dosimeters were not traced in this study. We therefore show the maximum possible error in Figure 4.

In cases of over-table tube irradiation, the eye lens is exposed much more than in cases of under-table tube irradiation because a considerable amount of diagnostic energy photons backscatter in the human body. Comparing the over-table tube and under-table tube situations, we observed that the under-table tube decreased the eye lens dose to one twenty-fourth. Most institutions conduct IR operations with the under-table tube, but some institutions still employ an over-table tube. In other words, the over-table tube approaches the threshold in an equivalent dose limit for an eye lens 24 times earlier, at most. Our results confirmed that exposure with the under-table tube can be highly beneficial for radioprotection.

The eye lens dose can differ drastically depending on the amount of lead protection. Comparison of over-table tube irradiation that occurred with and without a suspended lead screen showed that the lead screen reduced the eye lens dose to one-twelfth of the dose without the screen. Results demonstrated that $46 \mathrm{~h}$ of exposure in the over-table at a horizontal distance of $85 \mathrm{~cm}$ from the X-ray source at a height of $150 \mathrm{~cm}$ from the floor tube reached the ICRP eye lens dose limit (20 mSv/year) [28]. Use of the suspended lead screen extended the limit to 552 h. Furthermore, lead glass reportedly reduces the eye lens dose to about one-tenth of the usual dose without glass [29]. With VR-DVS simulating a realistic experience, visualization of dose distributions based on the physical process using Monte Carlo simulation by FDEIR can be expected to produce an instinctive understanding in users of the effects of protection.

Actually, FDEIR and VR-DVS can accommodate calculations for any IR room. In this study, the geometry was based on our institution's IR room using one fluoroscopic condition, but the results are expected to be useful for training and education. Intuitively, users can grasp the spread of the dose from the X-ray source. When building a VR space tailored to other facilities, the simulation geometry and virtual IR room can be reconstructed and the doses recalculated with less effort, for example, merely by tuning some parameters for room size. In addition, FDEIR works on a retail-grade GPU on a personal computer as well as on a supercomputing system. For instance, one GPU (GeForce GTX 1080; NVIDIA Corp.) on a personal computer took longer than $300 \mathrm{~h}$ for the necessary computations to support the simulations used for this study compared to $40 \mathrm{~h}$ on a supercomputing system.

Fast air dose simulation by FDEIR presents several salient benefits. First, fast air dose calculation using FDEIR eliminates the time-consuming measurements that are currently necessary for typical methods to map the dose distributions in the IR room. Air dose measurements demand great effort to install numerous dosimeters throughout a room, with repeated irradiation necessary for each condition. The fast and easy simulation method described herein provides an air dose distribution of approximately $10 \%$ difference in a few hours. Secondly, the alterable geometry and fast simulation are useful for radiation shielding calculations. Room configurations, especially lead shielding and the patient's body, affect the air dose distribution. By reconstructing the simulation geometry of an IR room, the air dose distributions can be estimated for widely diverse geometries and conditions. According to earlier reports, the suspended lead screen reduces the eye lens dose by 1.3-33 times depending on the geometry and conditions [29]. The use of FDEIR can simplify dose calculations for any institution.

Any location with sufficient space and a computer can be used to train medical staff in VR-DVS. This flexibility is expected to be particularly beneficial in cases with limited opportunities to use an actual apparatus [30-32]. Furthermore, dose visualization using VR technology applies to other modalities such as radiography, CT and radiotherapy. For clinical use, mixed reality and AR technology, which superimpose a virtual object on a real object, are expected to be more useful. Results of VR-DVS present the possibility of expansion to mixed reality and AR systems.

In conclusion, this study presented an air dose estimation technique using FDEIR and VR-DVS. Rapid dose estimation using GPU enabled calculation of the air dose easily under diverse conditions. Based on these results, VR-DVS is expected to open new horizons for education about radiation protection in IR.

Acknowledgement: This work was partly supported by a Japan Society for the Promotion of Science (JSPS) KAKENHI Grant (No. 18K07646).

Conflict of interest: The authors declare that they have no conflicts of interest to report.

This study has been presented at RSNA2017, $115^{\text {th }}$ JSMP, and $47^{\text {th }}$ JSIR. 
Disclaimer: Hiroshi Kondo is one of the Editorial Board members of Interventional Radiology. This author was not involved in the peerreview or decision-making process for this paper.

\section{References}

1. Sánchez RM, Vano E, Fernández JM, Rosales F, Sotil J, Carrera F, et al. Staff Doses in Interventional Radiology: A National Survey. J Vasc Interv Radiol 2012; 23: 1496-1501.

2. Pantos I, Patatoukas G, Katritsis DG, Efstathopoulos E. Patient radiation doses in interventional cardiology procedures. Curr Cardiol Rev 2009; 5: 1-11.

3. ICRP. Education and Training in Radiological Protection for Diagnostic and Interventional Procedures. ICRP Publication 113. Ann ICRP 2009; 39(5).

4. Friedman AA, Ghani KR, Peabody JO, Jackson A, Trinh QD, Elder JS. Radiation safety knowledge and practices among urology residents and fellows: Results of a nationwide survey. J Surg Educ 2013; 70: 224-231.

5. Chambers CE, Fetterly AK, Holzer R, Paul Lin P-J, Blankenship JC, Balter S, et al. Radiation safety program for the cardiac catheterization laboratory. Catheter Cardiovasc Interv 2011; 77: 546556.

6. Park PE, ParkJM, Kang JE, Cho JH, Cho SJ, Kim JH, et al. Radiation safety and education in the applicants of the final test for the expert of pain medicine. Korean J Pain 2012; 25: 16-21.

7. Bott OJ, Wagner M, Duwenkamp C, Hellrung N, Dresing K. Improving education on $\mathrm{C}$-arm operation and radiation protection with a computer-based training and simulation system. Int J Comput Assist Radiol Surg 2009; 4: 399-407.

8. Loy Rodas N, Padoy N. Seeing is believing: increasing intraoperative awareness to scattered radiation in interventional procedures by combining augmented reality, Monte Carlo simulations and wireless dosimeters. Int J Comput Assist Radiol Surg 2015; 10: 1181-1191.

9. Loy Rodas N, Barrera F, Padoy N. See It with Your Own Eyes: Markerless Mobile Augmented Reality for Radiation Awareness in the Hybrid Room. IEEE Trans Biomed Eng 2017; 64: 429-440.

10. Xiong Z, Guo C, Kilian-Meneghin J, Rudin S, Bednarek DR. Evaluation of methods of displaying the real-time scattered radiation distribution during fluoroscopically guided interventions for staff dose reduction. Medical Imaging 2018: Physics of Medical Imaging 2018; 1057366.

11. Chan LW, Chan T, Cheung BT, Mo K, Fung KK. Simulation, visualization and dosimetric validation of scatter radiation distribution under fluoroscopy settings. J Biomed Eng Informatics 2015; 1: $93-102$

12. Seymour NE, Gallagher AG, Roman SA, O’Brien MK, Bansal VK, Andersen DK, et al. Virtual reality training improves operating room performance: results of a randomized, double-blinded study. Ann Surg. 2002; 236: 454-458.

13. Gallagher AG, Ritter EM, Champion H, Higgins G, Fried MP, Moses G, et al. Virtual reality simulation for the operating room: Proficiency-based training as a paradigm shift in surgical skills training. Ann Surg 2005; 241: 364-372.

14. Takata $\mathrm{T}$, Kotoku J, Maejima H, Kumagai $\mathrm{S}$, Arai N, Kobayashi T, et al. Fast skin dose estimation system for interventional radiology. J Radiat Res 2018; 59: 233-239.

15. Badal A, Zafar F, Dong H, Badano A. A real-time radiation dose monitoring system for patients and staff during interventional fluoroscopy using a GPU-accelerated Monte Carlo simulator and an automatic 3D localization system based on a depth camera. Medical Imaging 2013: Physics of Medical Imaging 2013; 866828.
16. Sato T, Niita K, Matsuda N, Hashimoto $S$, Iwamoto $Y$, Noda $S$, et al. Particle and heavy ion transport code system, PHITS, ver. 2.52. J Nucl Sci Technol 2013; 50: 913-923.

17. Hirayama H, Namito Y, Bielajew AF, Wilderman SJ, Nelson WR. The EGS5 Code System. SLAC-R-730 and KEK Report 2005-8 (Stanford Linear Accelerator Center, Stanford, CA). 2005.

18. Mohammadi A, Baba M, Oishi T, Yamaguchi Y, Hagiwara M. Measurement and Calculation of Compton Spectrometer Response Function for 10-60-keV Monoenergetic Photons. J Nucl Sci Technol 2010; 47: 570-574.

19. Kajimoto T, Endo S, Tat Thanh N, Shizuma K. Calculation of coincidence summing in gamma-ray spectrometry with the EGS5 code. Appl Radiat Isot 2015; 95: 53-58

20. Berger MJ, Coursey JS, Zucker MA. ESTAR, PSTAR, and ASTAR: Computer Programs for Calculating Stopping-Power and Range Tables for Electrons, Protons, and Helium Ions (ver. 1.21) [Internet]. 1999. Available: http://physics.nist.gov/Star (1 September 2019, date last accessed).

21. L'Annunziata MF. Radiation Physics and Radionuclide Decay. Handbook of Radioactivity Analysis. Third ed. Elsevier; 2012. p.1162.

22. Zubal G, Harrell CR, Smith EO, Rattner Z, Gindi G, Hoffer PB. Computerized three-dimensional segmented human anatomy. Med Phys 1994; 21: 299-302.

23. ICRP. Conversion Coefficients for use in Radiological Protection against External Radiation. ICRP Publication 74. Ann ICRP 1996; 26(3-4).

24. Hart SG, Staveland LE. Development of NASA-TLX (Task Load Index): Results of Empirical and Theoretical Research. Adv Psychol 1988; 52: 139-183.

25. Lee K, Lee KM, Park MS, Lee B, Kwon DG, Chung CY. Measurements of Surgeons' Exposure to Ionizing Radiation Dose During Intraoperative Use of C-Arm Fluoroscopy. Spine (Phila PA 1976) 2012; 37: 1240-1244.

26. Grier RA. How high is high? A meta-analysis of nasa-tlx global workload scores. Proc. Hum. Factors Ergon. Soc. Annu. Meet 2015; 59(1): 1727-1731.

27. Chiyoda Technol. Corp. [Internet] Fluorescence glass dosimeter system (in Japanese) [cited 2019 Nov 20]. Available from: http:// isotope.c-technol.co.jp/pdf/DoseAce1407ATG.pdf

28. ICRP. ICRP Statement on Tissue Reactions / Early and Late Effects of Radiation in Normal Tissues and Organs - Threshold Doses for Tissue Reactions in a Radiation Protection Context. ICRP Publication 118. ICRP Publ 118 Ann ICRP 2012; 41(1/2).

29. Carinou E, Ferrari P, Bjelac OC, Gingaume M, Merce MS, O'Connor U. Eye lens monitoring for interventional radiology personnel: Dosemeters, calibration and practical aspects of $\mathrm{Hp}(3)$ monitoring. A 2015 review. J Radiol Prot 2015; 35: R17-R34.

30. Van Dam A, Laidlaw DH, Simpson RM. Experiments in Immersive Virtual Reality for Scientific Visualization. Comput Graph 2002; 26: 535-555.

31. King F, Jayender J, Bhagavatula SK, Shyn PB, Pieper S, Kapur T, et al. An Immersive Virtual Reality Environment for Diagnostic Imaging. J Med Robot Res 2016; 01: 1640003.

32. Kockro RA, Stadie A, Schwandt E, Reisch R, Charalampaki C, $\mathrm{Ng}$ I, et al. A Collaborative Virtual Reality Environment For Neurosurgical Planning And Training. Oper Neurosurg 2007; 61: 379391.

Interventional Radiology is an Open Access journal distributed under the Creative Commons Attribution-NonCommercial 4.0 International License. To view the details of this license, please visit (https://creativecommons.org/licenses/by$\mathrm{nc} / 4.0 /)$. 
Interventional Radiology 2020; 5: 58-66 